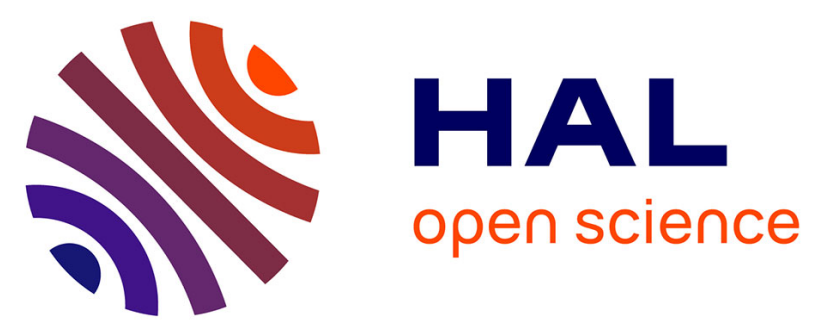

\title{
Homogames un jour, homogames toujours? Rencontre pendant les études et proximité de diplôme et de carrière au sein des couples en France
}

Milan Bouchet-Valat, Sébastien Grobon

\section{- To cite this version:}

Milan Bouchet-Valat, Sébastien Grobon. Homogames un jour, homogames toujours? Rencontre pendant les études et proximité de diplôme et de carrière au sein des couples en France. Population (édition française), 2019, D'un couple à l'autre: Numéro spécial autour de l'Étude des parcours individuels et conjugaux en France, 74 (1-2), pp.131-154. 10.3917/popu.1901.0131 . hal-02280967

\section{HAL Id: hal-02280967 \\ https://hal.science/hal-02280967}

Submitted on 6 Sep 2019

HAL is a multi-disciplinary open access archive for the deposit and dissemination of scientific research documents, whether they are published or not. The documents may come from teaching and research institutions in France or abroad, or from public or private research centers.
L'archive ouverte pluridisciplinaire HAL, est destinée au dépôt et à la diffusion de documents scientifiques de niveau recherche, publiés ou non, émanant des établissements d'enseignement et de recherche français ou étrangers, des laboratoires publics ou privés. 


\section{Milan BOUCHET-VALAT* et Sébastien GROBON**}

\section{Homogames un jour, homogames toujours? Rencontre pendant les études et proximité de diplôme et de carrière au sein des couples en France}

La massification scolaire et l'allongement des études en France depuis les années 1960 ont fortement affecté la mise en couple. Ces transformations expliquent, pour partie, le recul de l'âge à la première union (Galland, 1995; Prioux, 2003) et le développement des cohabitations hors mariage (VilleneuveGokalp, 1990). La littérature sur le choix du conjoint a porté une grande attention à ces évolutions, en étudiant, notamment, leurs effets sur l'homogamie d'éducation, soit la tendance à choisir un conjoint d'un niveau de diplôme proche ou identique au sien.

Partant du constat d'une augmentation de l'homogamie d'éducation aux États-Unis (Mare, 1991; Schwartz et Mare, 2005), certains auteurs (Blossfeld et Timm, 2003) ont vu dans l'allongement des études le premier facteur d'une série causale entraînant in fine un accroissement des inégalités entre ménages, susceptible de se transmettre d'une génération à l'autre. Selon cette théorie des "systèmes éducatifs comme marchés conjugaux », la massification scolaire renforce l'homogénéité des réseaux sociaux aux âges typiques de mise en couple et, ainsi, augmente la probabilité de rencontrer un conjoint du même niveau d'études que soi. Comme le diplôme fait partie des déterminants principaux du statut professionnel futur des individus, et donc de leurs revenus, une polarisation des ressources économiques, opposant couples favorisés et couples défavorisés, serait inévitable.

Ce raisonnement, en apparence implacable, souffre pourtant de deux faiblesses majeures. Tout d'abord, cette théorie se fonde sur une extrapolation

\footnotetext{
* Institut national d'études démographiques (Ined).

** Institut national de la statistique et des études économiques (Insee), Institut national d'études démographiques (Ined).

Correspondance : Milan Bouchet-Valat, Institut national d'études démographiques, 133, boulevard Davout, 75020 Paris. Courriel : milan.bouchet-valat@ined.fr
} 
réalisée principalement à partir de travaux portant sur les États-Unis. Mais, en Europe, l'homogamie d'éducation a, au contraire, nettement diminué depuis quelques décennies dans plusieurs pays, en particulier en France (voir BouchetValat (2014) pour une revue de littérature). La seconde limite tient à l'effet, supposé mécanique, de l'homogamie d'éducation sur le renforcement des inégalités de revenus entre ménages. Or il est désormais établi que ces deux phénomènes n'entretiennent qu'un lien relativement lâche (Worner, 2006; Breen et Salazar, 2010, 2011; Breen et Andersen, 2012; Eika et al., 2014; Frémeaux et Lefranc, 2015; Courtioux et Lignon, 2015), ce qui pourrait tenir au fait que l'homogamie de diplôme ne garantirait pas qu'au bout de quelques années les conjoints occupent des positions professionnelles plus proches que la moyenne.

Cet article s'intéresse au lien entre l'allongement des études et les inégalités de revenus en analysant, d'une part, le contexte de rencontre des conjoints au fil des générations et son interaction avec l'homogamie de diplôme et, d'autre part, les différences de carrière professionnelle au sein des couples. Premièrement, dans quelle mesure la massification scolaire s'est-elle traduite par un développement des mises en couple durant les études et, en particulier, de celles qui résultent d'une rencontre dans le cadre scolaire ou étudiant? Deuxièmement, les couples formés dans ce contexte se révèlent-ils plus homogames que les autres, qu'il s'agisse du diplôme ou de l'origine sociale? Troisièmement, l'homogamie au début de la relation présente-t-elle des effets durables sur les positions professionnelles des conjoints, en dépit des inégalités dans le déroulement des carrières des femmes et des hommes?

L'enquête Étude des parcours individuels et conjugaux (Épic, Ined-Insee, 2013-2014) offre une occasion inédite d'appréhender ces questions pour le cas français, grâce, à la fois, à des questions détaillées sur les lieux de rencontre et à une approche biographique, permettant une étude longitudinale des carrières professionnelles des conjoints.

\section{Revue de littérature}

\section{Rencontres pendant les études et évolution des lieux de rencontre}

La littérature sociologique sur la formation des couples a mis en évidence une évolution des circonstances de la rencontre du conjoint au fil des cohortes de premières unions. En France, Michel Bozon et François Héran (1987) ont relevé une diversification des lieux de rencontre et un net recul des rencontres dans le voisinage, entre le début du XX $x^{e}$ siècle et les années 1980. Le bal (ou la fête publique) a connu son apogée dans les années 1960, avant de décliner au profit des boîtes de nuit, qui ont culminé dans les années 1980, et des fêtes entre amis. 
L'augmentation marquée des rencontres dans le cadre des études constitue l'évolution la plus notable depuis les années 1960 : à cette époque, 8 \% des hommes et $5 \%$ des femmes trouvaient ainsi leur premier conjoint, contre respectivement 18 \% et 15 \% dans les années 1990 (Bozon et Rault, 2012). Cependant, ce cadre est loin d'être majoritaire, même si nous ne pouvons exclure que des rencontres survenues dans d'autres situations, telles que les soirées entre amis, soient en fait liées à la sociabilité étudiante.

Par ailleurs, les rencontres dans des lieux ouverts, c'est-à-dire, suivant la typologie de Michel Bozon et François Héran (1988), des lieux n'imposant aucune sélection à l'entrée, que ce soit dans un contexte de danse (bal, fête publique, boîte de nuit) ou dans un autre cadre (voisinage, lieu public, annonce), demeurent fréquentes, même si elles sont en baisse (leur part est passée de $55 \%$ pour les cohortes de mise en couple 1960-1968 à $34 \%$ pour les cohortes 1991-1998, selon Michel Bozon et Wilfried Rault). Des tendances similaires ont été relevées en Grande-Bretagne (Lampard, 2007).

\section{La rencontre dans le cadre des études a-t-elle une influence sur l'homogamie?}

Il peut sembler inévitable que les couples formés dans le cadre des études soient homogames en matière de diplôme, tant les lieux réservés que constituent les établissements scolaires ou universitaires sélectionnent, par définition, leur public sur ce critère. Il est pourtant moins évident qu'il n'y paraît que les conjoints qui se sont rencontrés sur les bancs de l'école ou de l'université obtiennent nécessairement les mêmes diplômes : leurs études peuvent, par exemple, se prolonger ou s'interrompre prématurément. En particulier, pour les générations les plus anciennes, les rôles d'étudiante et d'épouse pouvaient sembler incompatibles au vu des normes sociales dominantes (Singly, 1987). De surcroît, bien que le lien entre la rencontre pendant les études et l'homogamie de diplôme figure en bonne place dans l'influente théorie des systèmes éducatifs comme marchés conjugaux (Blossfeld et Timm, 2003), la littérature à ce sujet est assez peu développée.

Concernant la France, Michel Bozon et François Héran (1988) ont observé que les femmes diplômées de l'enseignement supérieur qui ont rencontré leur conjoint à l'université se sont un peu plus souvent mises en couple avec un fils de cadre que les autres (38\% contre $31 \%$ ), et plus souvent encore avec un futur cadre (dans $60 \%$ des cas, contre $43 \%$ pour les autres modes de rencontre). Les rencontres liées aux études semblent donc renforcer l'homogamie. À l'inverse, les rencontres survenues dans des lieux atypiques ${ }^{(1)}$ pour un groupe social sont elles-mêmes atypiques, et donc peu homogames. Les enfants de cadre qui ont rencontré leur conjoint dans un lieu ouvert ont choisi deux fois moins souvent des enfants de cadre que les autres; inversement, les enfants d'ouvrier qui ont rencontré leur

(1) Au sens où les membres de ce groupe social y rencontrent peu fréquemment leur conjoint (terme utilisé par Bozon et Héran). 
conjoint dans un lieu fermé ont choisi des enfants de cadre deux fois plus souvent que les autres pour les filles, et trois fois plus souvent pour les garçons.

Plus récemment, Marie Bergström (2016) a montré à partir des données de l'enquête Épic que les couples qui se sont rencontrés sur Internet sont moins homogames en matière d'éducation que ceux qui se sont rencontrés dans le cadre des études ou du travail, à caractéristiques de l'union contrôlées ${ }^{(2)}$. En revanche, ils ne se différencient pas du point de vue de l'homogamie en matière de profession et d'origine sociale.

Quelques études sont disponibles pour d'autres pays que la France. L'une d'elles, portant sur les États-Unis, a relevé que les couples (mariés ou non, cohabitants ou non) qui se sont rencontrés à l'école ou à l'université sont un peu plus homogames en matière d'éducation que les autres (Laumann et al., 1994). De leur côté, Matthijs Kalmijn et Henk Flap (2001) ont montré à partir de données néerlandaises que les conjoints qui ont fréquenté le même établissement scolaire sont plus homogames que les autres en termes de diplôme, en contrôlant des effets de structure à l'aide de modèles log-linéaires. Les autres contextes de rencontre (travail, associations, famille, voisinage) n'ont pas d'effet statistiquement significatif sur l'homogamie d'éducation, ce qui pourrait en partie tenir à la faible taille de l'échantillon utilisé. Cette absence d'effet se retrouve en ce qui concerne l'homogamie d'origine sociale. Enfin, Gina Potarca (2017) a également confirmé, à l'aide de modèles log-linéaires, qu'aux ÉtatsUnis et en Allemagne, les rencontres survenues dans le cadre des études étaient celles qui donnaient lieu à la plus forte homogamie d'éducation.

Si les rencontres dans le cadre des études semblent donc bien associées à une plus grande proximité de diplômes entre conjoints, ce résultat n'a pas été clairement établi dans le cas français. Nous chercherons dans cet article à analyser s'il est valable pour toutes les cohortes de naissance, à la fois pour l'homogamie d'éducation et pour celle d'origine sociale.

\section{Les carrières des conjoints, toujours plus favorables pour les hommes?}

L'homogamie de diplôme ne suffit pas à elle seule à déterminer la proximité ou la distance entre les professions des conjoints tout au long d'une relation, car ils peuvent suivre des parcours assez différents, même s'ils ont obtenu le même diplôme. Ces divergences tiennent, notamment, au fonctionnement genré du marché du travail et à l'investissement différencié des femmes et des hommes dans leur carrière.

D'une part, les femmes tirent un moins bon rendement professionnel de leur diplôme que les hommes (Mainguené et Martinelli, 2010). D’autre part, elles ont plus fréquemment tendance à travailler à temps partiel ou à interrompre leur

(2) Précisément : âge en début de relation, année de début de relation, rang de la relation et catégorie socioprofessionnelle du répondant. 
activité professionnelle, en particulier après la naissance d'un enfant (Couppié et Épiphane, 2007; Meurs et al., 2010). Ainsi, même à caractéristiques du premier emploi contrôlées, les écarts de rémunération entre hommes et femmes s'accroissent en cours de carrière (Le Minez et Roux, 2001, 2002; Dupray et Moullet, 2005).

Ces phénomènes se traduisent au sein du couple par de meilleures carrières pour les hommes que pour leurs conjointes, c'est-à-dire par une tendance à l'hypergamie féminine en matière de statut professionnel ou de salaire (GuichardClaudic et al., 2009; Bouchet-Valat, 2017), qui contraste avec la supériorité des diplômes des femmes (Bouchet-Valat, 2015). Cette tendance ne s'inverse que lorsque le niveau d'éducation de la femme est nettement supérieur à celui de son conjoint ou que la carrière de ce dernier connaît des difficultés (Testenoire, 2008; Guichard-Claudic et al., 2009). La persistance de représentations qui assignent les femmes à la sphère privée et les hommes à la sphère professionnelle participe à la perpétuation de ces normes de comportement genrées, malgré un recul global de l'idée selon laquelle les femmes seraient naturellement moins compétentes professionnellement (Burricand et Grobon, 2015).

La prise en compte de ces inégalités au sein du couple est essentielle pour appréhender les carrières respectives des conjoints et mesurer l'évolution des différences de statut professionnel (hétérogamie professionnelle) au fil de la vie en couple. Dans la mesure où les positions professionnelles, et donc les revenus, des femmes sont en moyenne inférieurs à ceux de leurs conjoints, même à diplôme égal, l'homogamie de diplôme pourrait avoir un effet moins fort qu'escompté sur les inégalités entre couples.

\section{Données et méthodes}

Cet article s'appuie sur les données de l'enquête Étude des parcours individuels et conjugaux (Épic), réalisée par l'Ined et l'Insee en 2013-2014 en France métropolitaine. Un échantillon représentatif d'individus âgés de 26 à 65 ans a été interrogé sur chacune de ses « relations de couple ou relations amoureuses importantes », en cours ou passées ${ }^{(3)}$. Au total, 14699 relations ont été décrites par les 7825 personnes qui ont répondu à l'enquête. Épic est la seule source française récente comportant une description détaillée des lieux de rencontre ainsi que des renseignements sur la situation en début d'union (notamment la profession des deux conjoints), ce qui autorise une approche longitudinale de l'homogamie.

\section{Définition des variables}

Les relations étudiées dans l'enquête Épic, comme dans l'ensemble de l'article, correspondent aux « relations de couple ou relations amoureuses importantes ». Cette formulation permet d'inclure une diversité d'unions, sans que l'enquête

(3) Voir la présentation de l'enquête Épic publiée dans ce même volume de Population pour plus de précisions sur cette source (Rault et Régnier-Loilier, 2019). 
n'impose une définition restrictive du couple. Elle est susceptible d'être interprétée de manière variable selon les répondants. On peut penser que les plus jeunes accorderont plus d'importance à leurs premières relations, même éphémères, que les plus âgés. Cela peut nous amener à surestimer l'augmentation de la proportion de premières relations formées dans le cadre des études (sous-estimation dans les cohortes anciennes et surestimation dans les plus récentes).

Nous considérons indifféremment les relations hétérosexuelles cohabitantes et non cohabitantes, qu'elles aient ou non un statut légal. Dans Épic, les relations non cohabitantes représentent $48 \%$ des premières relations amoureuses et $56 \%$ des relations en cours depuis moins de cinq ans au moment de l'enquête, mais seulement $10 \%$ de l'ensemble des relations en cours à la date de l'enquête. Dans la mesure où nous nous intéressons aux circonstances de la rencontre, retenir tous les types d'unions permet de mieux capter le début des relations, sans préjuger de leur durée ${ }^{(4)}$. Les résultats de nos modèles ne sont pas modifiés si on se limite aux couples cohabitants.

Afin de mesurer la fréquence des mises en couple pendant les études, nous nous intéressons dans un premier temps à la fois aux premières relations et aux relations en cours. Le diplôme et l'origine sociale n'étant renseignés dans Épic que pour le conjoint au moment de l'enquête, nous nous concentrons ensuite sur les unions en cours. Ces relations sont, en moyenne, plus stables que les premières relations, et donc probablement plus homogames, les relations fortement hétérogames étant moins stables (Mäenpää et Jalovaara, 2014). Cette sélection n'est cependant pas un biais à proprement parler : les relations en cours correspondent à la réalité des unions à un instant donné, et ce sont elles qui déterminent, notamment, les inégalités entre couples.

Le cadre de la rencontre est recueilli par la question « Où vous êtes-vous rencontrés pour la première fois? ${ }^{(5)}$. Sept cadres sont distingués ici : Internet, lieux publics (bal, fête publique, foire, voisinage, rue, café, commerce...), loisirs (boîte de nuit, discothèque, lieu de vacances, concert, association), études (école, collège, lycée, université), travail, amis (fête entre amis, domicile privé), famille (réunion ou fête de famille). En complément de la fréquence des rencontres ayant eu lieu dans le cadre des études stricto sensu, nous avons calculé la fréquence des relations qui ont commencé alors que le répondant poursuivait ses études, en comparant la date de début de la relation avec celle de la fin des études.

L'homogamie lors de la rencontre est tout d'abord appréhendée selon deux dimensions, les diplômes des conjoints d'une part, leurs origines sociales d'autre part. Les diplômes des conjoints sont classés en sept catégories : aucun diplôme; BEPC; CAP ou BEP ; baccalauréat général, technologique ou professionnel; $\mathrm{Bac}+2 ; \mathrm{Bac}+3$ ou $\mathrm{Bac}+4 ; \mathrm{Bac}+5$ et supérieur. Leurs origines

(4) C'est aussi la perspective retenue par l'enquête Épic qui renseigne sur les caractéristiques des conjoints au début de la relation et non de la cohabitation.

(5) Un peu moins de $3 \%$ des individus ont déclaré un second cadre de rencontre. Seul le premier a été retenu. 
sociales sont approchées par la catégorie socioprofessionnelle (PCS) du père ${ }^{(6)}$ (en six postes : agriculteurs; commerçants, artisans et chefs d'entreprise; cadres et professions intellectuelles supérieures; professions intermédiaires; employés; ouvriers). Pour chacune de ces deux dimensions, l'homogamie est définie comme l'appartenance des deux conjoints à la même catégorie.

Pour étudier les carrières des conjoints, l'écart entre leurs professions respectives est mesuré à l'aide d'un indicateur continu fondé sur l'évaluation du prestige de ces dernières. Nous utilisons l'échelle élaborée par Christine Chambaz, Éric Maurin et Constance Torelli (1998), qui se base sur les catégories socioprofessionnelles (PCS) en 16 postes $^{(7)}$ : plus son score est élevé, plus une profession est valorisée socialement. Les scores ont été normalisés pour s'étaler de 0 à 100, des ouvriers non qualifiés aux professions libérales.

Deux indicateurs différents peuvent être calculés à l'aide de ce score. Nous recourons, d'une part, à un indicateur d'hétérogamie (c'est-à-dire l'inverse de l'homogamie), qui mesure la distance entre conjoints : il consiste à évaluer dans quelle mesure les positions des deux conjoints sont éloignées, sans tenir compte du sens de cet écart (en faveur de l'homme ou de la femme). D'un point de vue mathématique, il s'agit de la valeur absolue de la différence de score entre les conjoints, qui varie de 0 (pour des conjoints occupant des professions de prestige égal) à 100 (pour un.e ouvrier.ère non qualifié.e en couple avec un.e membre des professions libérales).

D'autre part, nous calculons un indicateur d'hypergamie, qui mesure l'écart genré entre conjoints : il est égal à la différence entre la position de l'homme et celle de la femme. On peut ainsi observer si les positions des hommes ou celles des femmes sont plus élevées que celles de leur conjoint. Cet écart varie de - 100 (pour un homme ouvrier non qualifié en couple avec une femme profession libérale) à +100 (pour un homme profession libérale en couple avec une femme ouvrière non qualifiée), en passant par 0 lorsque les conjoints ont des professions de même niveau de prestige.

\section{Champ et méthodes de l'analyse longitudinale}

L'analyse longitudinale de l'évolution de l'écart de score de profession au sein du couple repose sur le sous-échantillon des relations en cours au moment de l'enquête dans lesquelles chaque conjoint avait déjà travaillé au début de la relation (soit 53 \% des relations en cours au moment de l'enquête). Nous excluons

(6) La question portait sur la « profession principale». En cas d'ambiguïté, les enquêteurs avaient pour instruction de garder « celle à laquelle il a consacré le plus de temps ». En cas de non-réponse (soit $10 \%$ des cas), la profession de la mère a été retenue.

(7) Construite à partir du classement sur une échelle de 1 à 5 de 122 intitulés de professions réalisé par un échantillon d'individus interrogés par l'Insee en France en 1996, elle reflète « l'évaluation sociale des professions ». Elle appartient à la famille des échelles de prestige, par opposition à celles de position socioéconomique fondées sur des critères objectifs, comme le niveau de qualification ou le revenu. Les analyses des auteurs montrent que ces dimensions entrent dans les critères d'évaluation mobilisés par les personnes interrogées. 
ainsi les couples formés pendant les études de l'un des conjoints ${ }^{(8)}$. Parmi ceux retenus, $34 \%$ sont composés d'au moins une personne ne travaillant pas au moment de l'enquête. Nous les avons volontairement inclus dans le champ, en retenant le dernier emploi occupé. Nous considérons en effet que ces cas reflètent la réalité des interruptions de carrière, en particulier pour les femmes. Le retrait du marché du travail entraîne un ralentissement de carrière et le dernier emploi occupé représente une approximation (sans doute optimiste) de la position que l'individu pourrait occuper s'il cherchait à nouveau un emploi.

Des modèles de régression linéaire sont estimés sur ce sous-échantillon afin de comprendre les mécanismes reliant l'écart de statut professionnel entre conjoints au moment de l'enquête (variable dépendante) aux diplômes des conjoints et à leur écart professionnel lors de la formation du couple (variables indépendantes). Une fois exclus les couples dans lesquels l'un des conjoints n'avait jamais travaillé au début de la relation, la non-réponse aux variables explicatives du modèle est de $6 \%$. En l'absence de données sur la profession des conjoints à la fin des unions qui ne sont plus en cours à l'enquête, la variable de durée du couple peut être affectée par une sélection selon l'âge de mise en couple ou l'année de naissance, par exemple, dans l'hypothèse où les couples les moins homogames se sépareraient plus fréquemment. C'est pourquoi le rang de la relation et l'année de naissance ont été ajoutés comme variables de contrôle (leur effet dans les modèles n'est cependant pas significatif).

Un biais, dont le sens est difficile à évaluer, peut résulter de la restriction de l'analyse aux personnes ayant déclaré une profession en début de relation. Ce champ exclut les relations commencées pendant les études de l'un des conjoints, c'est-à-dire celles formées à un jeune âge ou dans lesquelles les conjoints ont suivi des études longues. Afin de vérifier dans quelle mesure la sélection du sous-échantillon peut affecter les résultats, nous avons estimé une variante des modèles de régression en utilisant la modélisation en deux étapes d'Heckman. L'âge de mise en couple des deux conjoints a été utilisé comme instrument pour prédire l'inclusion ou non dans le sous-échantillon (soit le fait que les deux conjoints aient déjà travaillé au début de l'union). Ce test confirme les résultats obtenus sur notre sous-échantillon.

\section{De plus en plus de rencontres pendant et dans le cadre des études}

La première des trois étapes de la théorie des systèmes éducatifs comme marchés conjugaux postule le développement des rencontres dans le cadre des études ou pendant celles-ci (Blossfeld et Timm, 2003). Dans cette partie, nous mettons ce schéma à l'épreuve en comparant les premières relations aux relations en cours à la date de l'enquête.

(8) Les « petits boulots d'été » sont explicitement exclus du questionnaire de l'enquête Épic. 
La massification scolaire a connu deux étapes principales en France dans la seconde moitié $\mathrm{du} \mathrm{XX}^{\mathrm{e}}$ siècle : une première vague a concerné les cohortes nées à partir du début des années 1940, une seconde celles nées à partir du début des années 1970 (Chauvel, 1998). Cette évolution a entraîné un allongement des études bien plus marqué que le recul de l'âge à la première mise en couple (Robert-Bobée et Mazuy, 2005; Rault et Régnier-Loilier, 2015). Par conséquent, la proportion de premières relations commencées avant la fin des études ainsi que celle des rencontres dans le cadre des études ont augmenté très nettement au fil des cohortes (figure 1A). Alors que moins de deux premières relations sur dix commençaient avant la fin des études (quel que soit le cadre de la rencontre) pour les cohortes nées en 1950, c'est le cas de cinq sur dix pour les hommes nés en 1980 et même de six sur dix pour les femmes de cette cohorte (ces dernières se mettant en couple plus tôt que les hommes). Entre ces mêmes cohortes, la part des rencontres dans le cadre des études a aussi augmenté, passant d'une première union sur dix à deux sur dix pour les femmes et à trois sur dix pour les hommes. Leur fréquence est moindre chez les femmes, ces dernières se mettant en couple avec des conjoints en moyenne un peu plus âgés qu'elles $^{(9)}$. Ces tendances semblent se prolonger pour les cohortes plus récentes, mais il est possible que les personnes n'ayant pas encore vécu de relation

Figure 1. Proportion de relations commencées pendant les études et de rencontres dans le cadre des études (1948 à 1988) selon la cohorte de naissance et le sexe

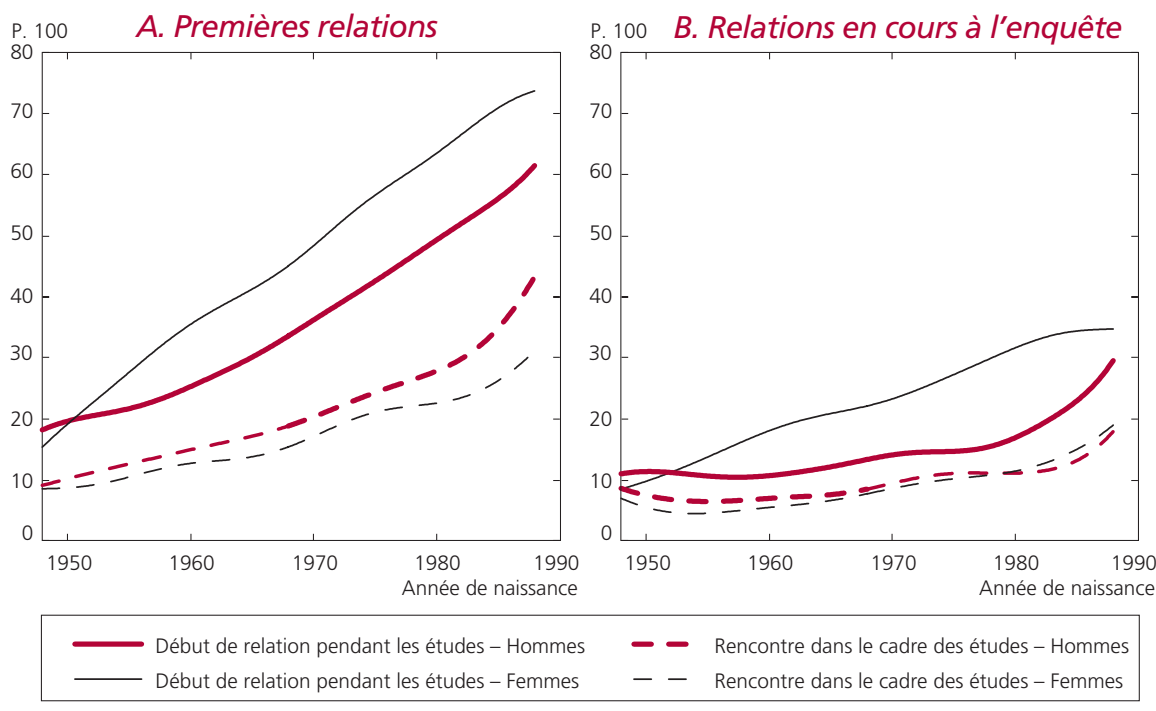

Champ : Relations de couple ou relations amoureuses « importantes» des personnes âgées de 26 à 65 ans vivant en France métropolitaine. Source : Épic (Ined-Insee, 2013-2014).

(9) En moyenne, toutes cohortes confondues, les premières conjointes des hommes ont 1,2 année de moins qu'eux et les premiers conjoints des femmes ont 2,7 années de plus qu'elles. 
cohabitante à la date de l'enquête donnent une définition plus large à la première relation importante, ce qui expliquerait cette apparente accélération.

Les séparations de certains couples au fil du temps atténuent cette tendance. Si l'on observe les relations en cours au moment de l'enquête (seules $45 \%$ d'entre elles sont des premières relations), la part des couples formés dans le cadre des études ou avant leur fin augmente au fil des cohortes, mais beaucoup plus faiblement (figure 1B). Une personne née en 1950 sur dix a rencontré son conjoint au moment de l'enquête pendant ses études, contre deux hommes sur dix et trois femmes sur dix né.e.s en 1980. Pour les mêmes cohortes, la part des rencontres dans le cadre des études passe d'un peu plus d'une sur vingt à une sur dix. Laugmentation observée au fil du temps pourrait ne refléter qu'un effet de l'âge : plus la personne interrogée appartient à une génération récente, plus elle est jeune à la date de l'enquête et susceptible de n'avoir connu qu'une seule union.

L'augmentation de la part de premières relations commencées dans le cadre des études n'est pas aussi importante que le laisserait penser la première étape du raisonnement de Hans-Peter Blossfeld et Andreas Timm. Dans la mesure où la part de rencontres au cours des études croît néanmoins au fil des générations, se pose la question de l'homogamie des couples formés dans ce contexte.

\section{Les rencontres dans le cadre des études favorisent l'homogamie éducative}

La seconde étape de la théorie des systèmes éducatifs comme marchés conjugaux repose sur l'hypothèse d'une plus forte homogamie de diplôme chez les couples qui se sont rencontrés dans le cadre des études. Si cette relation semble a priori triviale, nous avons vu qu'elle n'a que rarement fait l'objet d'une mise à l'épreuve empirique. Nous nous pencherons aussi sur l'homogamie d'origine sociale (c'est-à-dire de profession des pères), pour laquelle le lien avec les rencontres dans un cadre d'études paraît moins évident et qui constitue pour cela un point de comparaison intéressant. Lanalyse portera ici exclusivement sur les unions en cours à la date de l'enquête.

Comme attendu, les variations de l'homogamie selon le cadre de rencontre sont assez marquées (figure 2), en particulier celles de l'homogamie de diplôme, définie comme la proportion de couples dans lesquels les conjoints ont le même diplôme (axe horizontal). Alors que seulement 35 \% de l'ensemble des couples sont homogames, c'est le cas de $50 \%$ de ceux qui se sont rencontrés dans le cadre des études. À l'opposé, les relations qui ont débuté sur Internet ${ }^{(10)}$ et dans le cadre de loisirs s'avèrent les moins homogames, avec des taux respectifs de

(10) Les rencontres commencées sur Internet sont assez rares (4\% des unions en cours) et $87 \%$ d'entre elles sont des remises en couple. En contrôlant, entre autres, l'âge et l'année de début de relation, ainsi que le rang de l'union, la plus faible homogamie observée pour ce cadre de rencontre disparaît (Bergström, 2016). 
Figure 2. Homogamie de diplôme et d'origine sociale selon le lieu de rencontre des conjoints ayant une relation au moment de l'enquête

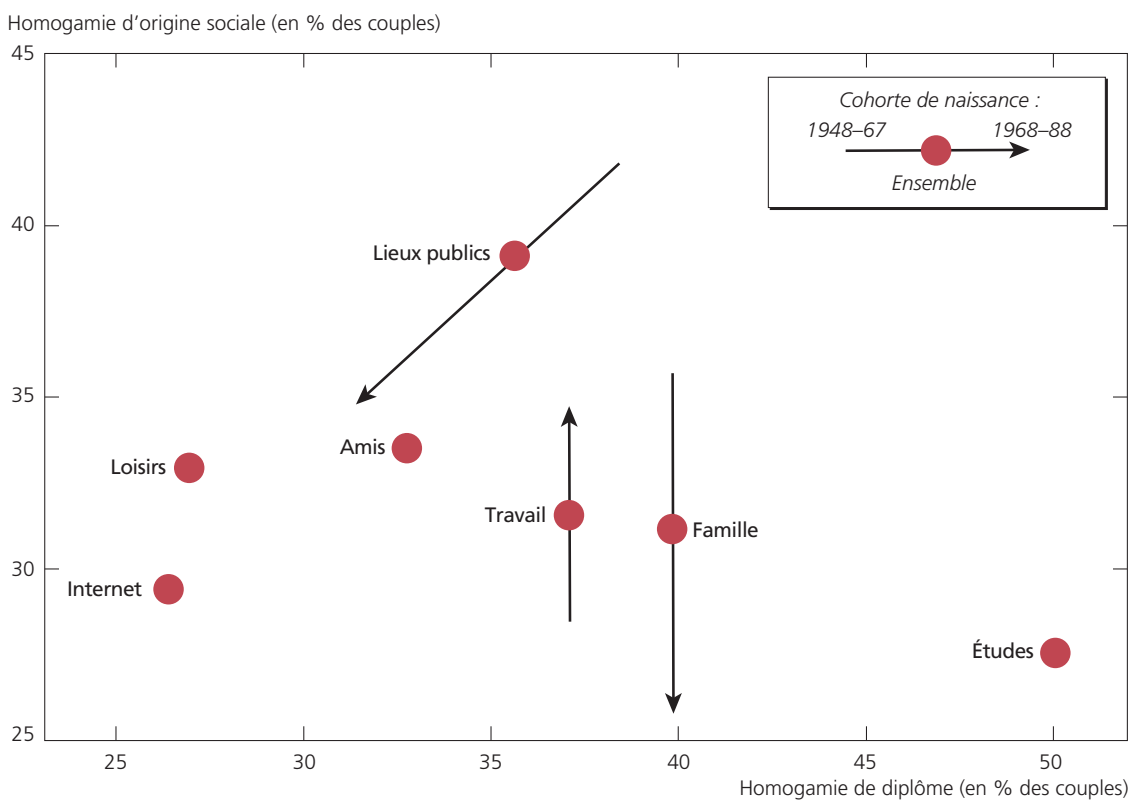

Lecture : Pour $39 \%$ des couples qui se sont rencontrés dans un lieu public, les pères des conjoints ont la même catégorie socioprofessionnelle; cette proportion atteint 42 \% pour les individus nés entre 1948 et 1967 et 35 \% pour ceux nés entre 1968 et 1988. Parmi ces mêmes couples, la proportion de ceux dans lesquels les conjoints ont le même diplôme est passée de $38 \%$ à $31 \%$ entre ces deux cohortes.

Note : Les flèches montrent l'évolution entre les deux cohortes lorsqu'elle est statistiquement significative au seuil de $5 \%$. Dans le cas contraire, seul le point moyen est indiqué.

Champ : Relations de couple ou relations amoureuses « importantes » en cours à la date de l'enquête (personnes âgées de 26 à 65 ans).

Source : Épic (Ined-Insee, 2013-2014).

$26 \%$ et de $27 \%$. Entre ces deux extrêmes, les rencontres par le biais des amis et de la famille, du travail ou dans des lieux publics sont proches de la moyenne.

Il est assez étonnant de constater que les variations de l'homogamie d'origine sociale (axe vertical) sont bien différentes de celles de l'homogamie de diplôme. Si les rencontres dans le cadre des études sont, de loin, les plus homogames du point de vue du diplôme, elles sont au contraire les moins homogames du point de vue de l'origine sociale ( $28 \%$ contre $33 \%$ de l'ensemble des rencontres). À l'inverse, les rencontres dans des lieux publics, pour lesquelles l'homogamie d'éducation se situe dans la moyenne, présentent l'homogamie d'origine sociale la plus forte (39\%). Les rencontres dans le cadre des loisirs entraînent aussi une faible homogamie de diplôme, mais une homogamie d'origine sociale dans la moyenne. Contrairement à ce que l'on aurait pu supposer a priori, les rencontres dans le cadre des études ne favorisent donc pas systématiquement l'homogamie.

Des analyses complémentaires ont été réalisées à l'aide de modèles visant à contrôler l'effet de structure lié aux caractéristiques sociales des individus 
qui se rencontrent dans chacun de ces contextes ${ }^{(11)}$. L'homogamie d'origine sociale, tout d'abord, se révèle moindre chez les couples qui se sont rencontrés dans le cadre de leurs études uniquement parce que cette population est plus hétérogène. Les enfants de cadres (groupe de faible taille) y sont sur-représentés et les enfants d'ouvriers et d'employés (groupes de grande taille) sous-représentés : la relative diversité des origines sociales augmente mécaniquement la probabilité de former un couple hétérogame à cet égard. On observerait un niveau d'homogamie similaire si les couples qui se sont rencontrés dans ce cadre étaient formés au hasard, par échange aléatoire des conjoints au sein de ce groupe. En revanche, la plus forte homogamie éducative des couples qui se sont rencontrés pendant leurs études ne s'explique pas uniquement par la composition de cette population. Dans ce cas, les diplômes des conjoints sont plus proches que si tous les individus qui se sont mis en couple pendant leurs études étaient appariés au hasard : une sélection supplémentaire s'opère donc selon le niveau d'éducation. Ces résultats sont cohérents avec les travaux étrangers cités précédemment (Kalmijn et Flap, 2001; Potarca, 2017).

L'étude des évolutions au fil des cohortes montre que les couples formés dans le cadre des études sont tout autant homogames dans les générations nées entre 1948 et 1967 que dans celles nées entre 1968 et $1988^{(12)}$. En tant que marché conjugal, le système éducatif ne favorise donc pas plus l'homogamie qu'avant la massification scolaire.

Le taux d'homogamie correspondant à d'autres cadres de rencontre a, en revanche, évolué. Tout d'abord, il s'est nettement affaibli pour les couples qui se sont rencontrés dans des lieux publics, passant de $38 \%$ à $31 \%$ pour le diplôme, et de 42 \% à 35 \% pour l'origine sociale. Cette baisse s'explique par le déclin du voisinage comme lieu de rencontre du conjoint, alors qu'il jouait un rôle essentiel, en particulier pour les classes populaires et les agriculteurs (Bozon et Héran, 1987), et favorisait une forte proximité sociale des conjoints (homogamie de $56 \%$ pour le diplôme et de $44 \%$ pour l'origine sociale pour la cohorte 1948-1967). Le même phénomène s'observe pour les rencontres dans le cadre familial : elles donnaient naissance à des couples homogames pour l'origine sociale dans $35 \%$ des cas pour les générations nées entre 1947 et 1967, mais dans seulement $26 \%$ des cas pour les générations nées entre 1968 et 1988. Cependant, aucun changement n'est visible concernant la proximité de diplôme.

(11) Il s'agit de modèles log-linéaires (ici un modèle log-multiplicatif à effet de couche ou UNIDIFF), utilisés dans plusieurs travaux antérieurs (Kalmijn et Flap, 2001 ; Potarca, 2017), dont ne sont résumées que les conclusions essentielles à titre de comparaison. Voir Potarca (2017) pour une présentation plus détaillée de la spécification. Cette approche s'appuie sur des données portant sur les lieux de rencontre, mais ne permet pas de contrôler leur fréquentation par des personnes qui ne s'y sont pas mises en couple (résultats détaillés disponibles sur demande auprès des auteurs et sur http://bouchet-valat.site.ined.fr).

(12) Les proportions calculées pour chacune des cohortes ne diffèrent pas significativement; les intervalles de confiance à $95 \%$ sont respectivement de [43\%;59\%] pour la première cohorte et de [43\%; $55 \%$ ] pour la seconde. 
À l'opposé, les rencontres dans le cadre professionnel menaient à une homogamie d'origine sociale dans $29 \%$ des cas pour la première cohorte et dans $35 \%$ des cas pour la cohorte plus récente. On peut interpréter cette hausse comme une conséquence de l'amélioration des carrières professionnelles des femmes : la mixité sexuelle des environnements de travail augmenterait la probabilité que surviennent des rencontres de niveau social égal. De fait, cette évolution a uniquement concerné les cadres et les professions intermédiaires.

Enfin, aucune différence statistiquement significative concernant l'hypergamie féminine, c'est-à-dire de tendance à un écart systématique en faveur de l'homme, n'a pu être relevée selon les cadres de rencontre. Les effectifs de l'enquête ne permettent pas de déterminer si, parmi les couples dans lesquels les conjoints ont des diplômes différents, la proportion de cas dans lesquels la femme est la plus diplômée varie selon le cadre de rencontre.

\section{Les effets de l'homogamie de diplôme sur les écarts de carrière professionnelle dans le couple}

Les rencontres en cours d'études renforcent donc l'homogamie de diplôme. La troisième et dernière étape de notre analyse vise à déterminer s'il existe un lien entre l'homogamie de diplôme, qui est donnée une fois pour toutes à la mise en couple, et la similarité des statuts professionnels. L'homogamie de diplôme conduit-elle à un rapprochement des professions des conjoints? Si c'est le cas, comment s'articule-t-elle avec l'écart professionnel à la mise en couple et avec les évolutions de carrière ultérieures?

Pour répondre à ces questions, nous étudions tout d'abord les variations de l'écart de profession entre les conjoints à la date de l'enquête selon leur proximité éducative. Puis nous cherchons à comprendre les mécanismes à l'œuvre par une approche longitudinale tenant compte de l'écart professionnel initial. Nous utilisons un score de prestige des professions (cf. partie II), afin de calculer, d'une part, une distance entre conjoints (indicateur d'hétérogamie, égal à la valeur absolue de l'écart de score), d'autre part, un écart genré (indicateur d'hypergamie, égal à la différence entre le score de l'homme et celui de la femme).

\section{Les statuts professionnels sont plus proches} lorsque les conjoints ont le même diplôme

Lécart professionnel à la date de l'enquête, qu'il soit calculé en termes de distance ou de genre, permet d'appréhender un point d'arrivée moyen résultant à la fois de l'écart professionnel à la mise en couple et des évolutions de carrière ultérieures des conjoints. Comme le prévoit la théorie de Blossfeld et Timm, la proximité de diplôme réduit la distance professionnelle (indicateur 
d'hétérogamie), toutes durées de vie en couple confondues (figure 3A). Lorsque les conjoints possèdent le même diplôme, la distance professionnelle à l'enquête est inférieure de 3 points de prestige à celle des couples dans lesquels la femme est la plus diplômée, et de 7 à 8 points par rapport à ceux dans lesquels l'homme est le plus diplômé (soit une distance respectivement inférieure de 14 \% et $30 \%$ par rapport à chaque référence) ${ }^{(13)}$. On n'observe pas de différence de génération sur ce point.

Les effets diffèrent nettement selon que l'homme ou la femme est plus diplômé.e et une analyse de l'écart genré de statut professionnel confirme l'importance des inégalités selon le sexe (figure 3B). Même quand les deux conjoints ont un niveau de diplôme identique, l'écart est en moyenne en faveur de l'homme (hypergamie) à la date de l'enquête, pour les générations les plus anciennes ( 9 points de prestige), comme pour les plus récentes ( 7 points). Il s'accentue lorsque l'homme est plus diplômé que sa conjointe (20 points), sans que l'on observe de différence entre générations. Et c'est lorsque la femme est la plus diplômée que l'écart genré de prestige professionnel à la date de l'enquête est le plus faible. Il est, en moyenne, en sa faveur, de l'ordre de 5 points de prestige pour les personnes nées depuis 1968, et de 1 point seulement pour celles nées avant cette date. Si l'écart de diplôme joue bien sur la proximité professionnelle des conjoints, le rendement professionnel des années d'études apparaît plus élevé pour les hommes que pour les femmes, en particulier pour les générations plus anciennes.

L'analyse des indicateurs d'hétérogamie et d'hypergamie à la date de l'enquête confirme que la distance professionnelle entre les conjoints varie selon la proximité de leurs diplômes, validant apparemment la troisième hypothèse de la théorie des systèmes éducatifs comme marchés conjugaux. Cependant, les effets observés restent d'une ampleur limitée. En outre, en matière de statut professionnel, la logique d'hypergamie féminine prévaut, y compris en cas d'homogamie de diplôme à la mise en couple, ce qui invite à étudier les évolutions de carrière des deux conjoints.

\section{Les différences de carrière entre conjoints dépendent peu des différences de diplômes}

Nous étudions à présent les mécanismes conduisant aux écarts de position professionnelle entre conjoints à la date de l'enquête afin de préciser les rôles respectifs de l'homogamie de diplôme et des inégalités entre les femmes et les hommes tout au long de la vie en couple.

Deux modèles de régression linéaire sont estimés dans un premier temps (A1 et A2 dans le tableau 1) pour expliquer l'hétérogamie à la date de l'enquête

(13) À titre d'indication, un écart de 10 points de prestige correspond à la différence entre le prestige des professions intermédiaires de l'enseignement, de la santé et de la fonction publique et celui des cadres de la fonction publique et des professions intellectuelles et artistiques; la différence entre ouvrier qualifié et ouvrier non qualifié correspond à 27 points. 
Figure 3. Moyennes de la distance et de l'écart genré de profession entre conjoints à la date d'enquête, selon la cohorte de naissance et le diplôme

\section{A. Distance (hétérogamie)}

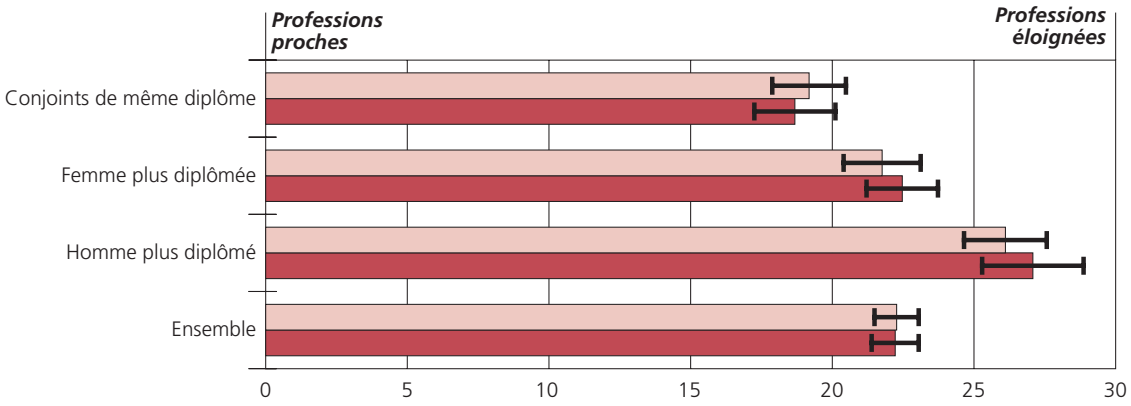

B. Écart genré (hypergamie)

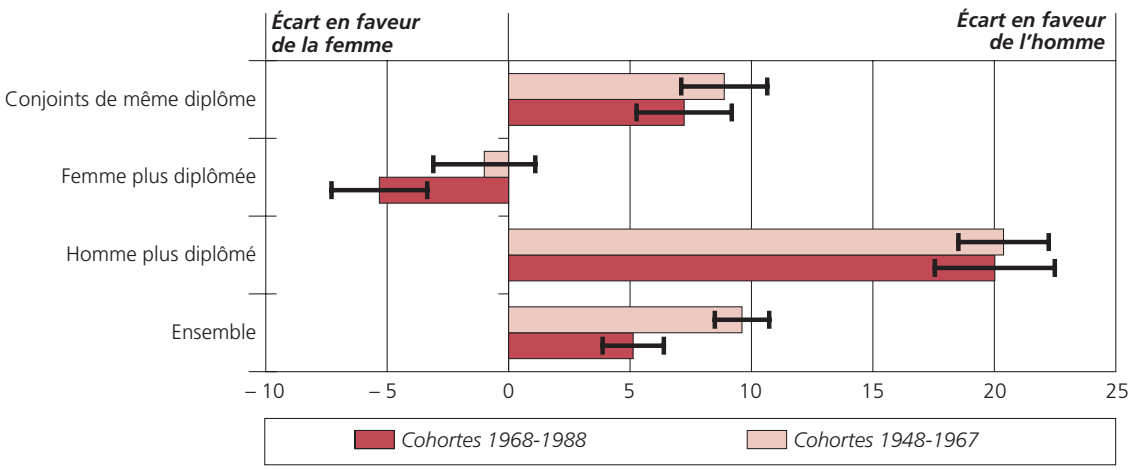

Lecture : Pour les cohortes nées à partir de 1968, lorsque les conjoints ont le même niveau de diplôme, la distance entre leurs professions (hétérogamie) à la date de l'enquête atteint, en moyenne, 19 points de prestige, sur une échelle de 0 à 100 .

Champ : Relations de couple ou relations amoureuses « importantes » en cours à la date de l'enquête et dans lesquelles les deux conjoints ont déjà travaillé (personnes âgées de 26 à 65 ans).

Source : Épic (Ined-Insee, 2013-2014).

(distance non genrée entre conjoints). L'analyse confirme l'effet de l'homogamie de diplôme sur la distance professionnelle entre conjoints, en contrôlant l'année de naissance, le rang de l'union, le statut d'activité des conjoints et le nombre d'enfants issus de la relation en cours (modèle A1). Mais le pouvoir explicatif de ce type d'homogamie est limité : seuls $2 \%$ de la variance $\left(\mathrm{R}^{2}\right)$ sont expliqués ${ }^{(14)}$. De surcroît, le pouvoir explicatif est le même si l'on modélise la distance professionnelle en début de relation et non plus à l'enquête, ce qui confirme le peu d'effet de l'homogamie de diplôme sur la position relative des conjoints, quelle que soit l'étape de la relation considérée. La faible ampleur de l'effet est également confirmée : selon cette modélisation, et à autres variables contrôlées, si tous les couples (et non plus un tiers de l'échantillon) devenaient homogames

(14) $7 \%$ en intégrant les 49 catégories qui décrivent de manière complète l'interaction entre diplômes des conjoints. 
en matière de diplôme, la distance moyenne entre conjoints passerait de 23 points à 20 points (soit une diminution de $13 \%$ seulement $)^{(15)}$.

Comme on pouvait s'y attendre, la distance professionnelle initiale apparaît bien plus déterminante pour expliquer la distance entre conjoints à la date d'enquête : la part de variance expliquée passe à $20 \%$ lorsque cette variable est ajoutée (modèle A2). Cependant, son influence demeure elle aussi partielle : pour des durées de vie en couple inférieures à 5 ans, $66 \%$ de la distance initiale se retrouvent à l'enquête. Cet effet décroît au fil des années de vie en couple $e^{(16)}$ (effets croisés allant de $-0,18$ de 5 à 14 ans, soit un effet total de $48 \%$, jusqu'à $-0,34$ pour plus de 35 ans, soit un effet total de $32 \%$ ). Ainsi, l'homogamie de profession est loin d'être donnée une fois pour toutes. Notons d'ailleurs que, même à distance professionnelle égale au début de la relation, les couples dans lesquels l'homme est plus diplômé présentent une distance plus importante au moment de l'enquête ( 7 points en moyenne). Cela s'explique par une tendance à l'accroissement de l'écart professionnel en faveur de l'homme, visible dans les modèles suivants.

Ces résultats se retrouvent dans l'analyse de l'hypergamie, autrement dit de l'écart genré entre la profession de l'homme et celle de la femme (modèles B1 et B2). La qualité d'ajustement du modèle aux données est bien meilleure lorsque l'on prend en compte les inégalités entre les femmes et les hommes: pour chaque type de modèle, la part de variance expliquée est près du double de celle obtenue pour l'explication de l'hétérogamie ( $\mathrm{R}^{2}$ de respectivement $12 \%$ pour le modèle $\mathrm{Bl}$ et $17 \%$ avec l'interaction complète entre diplômes des conjoints, et $35 \%$ en intégrant l'écart genré en début de relation, modèle B2).

À la différence du modèle précédent, le modèle genré (B2) met en évidence un effet de la durée de vie en couple, bien qu'il soit limité aux longues durées : les couples formés depuis plus de 25 ans sont caractérisés par un écart en faveur de l'homme plus élevé de 6 points que ceux formés depuis moins de 5 ans. Ce résultat semble indiquer un renforcement de l'hypergamie féminine au fil de la vie en couple. On ne peut cependant exclure qu'il reflète la plus forte hypergamie des couples formés avant 1990 (soit un effet de la cohorte de mise en couple, c'est-à-dire de la période à laquelle la relation a commencé, plutôt que de sa durée proprement dite).

En revanche, on retrouve un effet de l'écart professionnel du même ordre que dans le modèle non genré : pour une durée de vie en couple inférieure à 5 ans, $71 \%$ de l'écart professionnel en faveur de l'homme en début de relation se répercute sur l'écart à l'enquête, puis l'effet diminue au fil des années. D'autre part, l'effet des différences de diplôme dans le couple persiste également après

(15) Cette prédiction est obtenue en soustrayant à la distance moyenne observée les coefficients associés aux modalités « homme plus diplômé » et « femme plus diplômée » multipliés par la proportion que représentent ces groupes dans l'échantillon (soit $33 \%$ dans les deux cas).

(16) Malgré le contrôle de l'année de naissance dans le modèle, il n'est pas possible par définition de distinguer l'effet de la durée de vie en couple de celui de la période de mise en couple. 
Tableau 1. Facteurs explicatifs de I'hétérogamie et I'hypergamie professionnelles à l'enquête (régressions linéaires)

\begin{tabular}{|c|c|c|c|c|}
\hline & \multicolumn{2}{|c|}{ Distance (hétérogamie) } & \multicolumn{2}{|c|}{ Écart genré (hypergamie féminine ) } \\
\hline & A1 & $\mathrm{A} 2$ & B1 & B2 \\
\hline Constante & $\begin{array}{c}19,56 \\
(13,57 ; 25,54)\end{array}$ & $\begin{array}{c}20,22 \\
(14,64 ; 25,79)\end{array}$ & $\begin{array}{c}4,68 \\
(-3,60 ; 12,96)\end{array}$ & $\begin{array}{c}6,36 \\
(-0,54 ; 13,27)\end{array}$ \\
\hline \multicolumn{5}{|l|}{ Durée de la relation } \\
\hline$<5$ ans (Réf.) & 0 & 0 & 0 & 0 \\
\hline 5 à 14 ans & $\begin{array}{c}0,76 \\
(-2,27 ; 3,79)\end{array}$ & $\begin{array}{c}0,64 \\
(-1,87 ; 3,15)\end{array}$ & $\begin{array}{c}1,27 \\
(-3,01 ; 5,55)\end{array}$ & $\begin{array}{c}1,19 \\
(-2,06 ; 4,44)\end{array}$ \\
\hline 15 à 24 ans & $\begin{array}{c}1,14 \\
(-2,56 ; 4,83)\end{array}$ & $\begin{array}{c}1,56 \\
(-1,65 ; 4,76)\end{array}$ & $\begin{array}{c}0,68 \\
(-4,61 ; 5,98)\end{array}$ & $\begin{array}{c}0,04 \\
(-4,17 ; 4,26)\end{array}$ \\
\hline 25 à 34 ans & $\begin{array}{c}0,61 \\
(-3,84 ; 5,05)\end{array}$ & $\begin{array}{c}1,62 \\
(-2,38 ; 5,61)\end{array}$ & $\begin{array}{c}7,00 \\
(0,70 ; 13,30)\end{array}$ & $\begin{array}{c}5,79 \\
(0,63 ; 10,96)\end{array}$ \\
\hline 35 ans ou plus & $\begin{array}{c}1,30 \\
(-4,05 ; 6,65) \\
\end{array}$ & $\begin{array}{c}2,17 \\
(-2,85 ; 7,19)\end{array}$ & $\begin{array}{c}7,08 \\
(-0,33 ; 14,49) \\
\end{array}$ & $\begin{array}{c}6,44 \\
(0,27 ; 12,62)\end{array}$ \\
\hline \multicolumn{5}{|l|}{ Proximité éducative } \\
\hline $\begin{array}{l}\text { Conjoints de même } \\
\text { diplôme (Réf.) }\end{array}$ & 0 & 0 & 0 & 0 \\
\hline Homme plus diplômé & $\begin{array}{c}6,9 \\
(4,85 ; 8,96)\end{array}$ & $\begin{array}{c}5,05 \\
(3,19 ; 6,91)\end{array}$ & $\begin{array}{c}11,80 \\
(9,13 ; 14,47)\end{array}$ & $\begin{array}{c}6,36 \\
(3,98 ; 8,73)\end{array}$ \\
\hline Femme plus diplômée & $\begin{array}{c}1,86 \\
(-0,09 ; 3,81) \\
\end{array}$ & $\begin{array}{c}-0,02 \\
(-1,83 ; 1,79) \\
\end{array}$ & $\begin{array}{c}-10,87 \\
(-13,67 ;-8,07) \\
\end{array}$ & $\begin{array}{c}-5,61 \\
(-8,11 ;-3,12) \\
\end{array}$ \\
\hline $\begin{array}{l}\text { Distance de profession } \\
\text { en début de relation* }\end{array}$ & & $\begin{array}{c}0,66 \\
(0,55 ; 0,78)\end{array}$ & & \\
\hline $\begin{array}{l}\text { Écart genré de profession } \\
\text { en début de relation* }\end{array}$ & & & & $\begin{array}{c}0,71 \\
(0,62 ; 0,79)\end{array}$ \\
\hline \multicolumn{5}{|c|}{ Distance/écart initial·e $\times$ Durée de la relation } \\
\hline $\begin{array}{l}\text { Distance/écart initial.e } \times \\
<5 \text { ans (Réf.) }\end{array}$ & & 0 & & 0 \\
\hline $\begin{array}{l}\text { Distance/Écart initial·e } \times \\
5 \text { à } 14 \text { ans }\end{array}$ & & $\begin{array}{c}-0,18 \\
(-0,33 ;-0,04)\end{array}$ & & $\begin{array}{c}-0,12 \\
(-0,24 ;-0,01)\end{array}$ \\
\hline $\begin{array}{l}\text { Distance/écart initial·e × } \\
15 \text { à } 24 \text { ans }\end{array}$ & & $\begin{array}{c}-0,23 \\
(-0,39 ;-0,08)\end{array}$ & & $\begin{array}{c}-0,24 \\
(-0,37 ;-0,10)\end{array}$ \\
\hline $\begin{array}{l}\text { Distance/écart initial.e × } \\
25 \text { à } 34 \text { ans }\end{array}$ & & $\begin{array}{c}-0,28 \\
(-0,44 ;-0,11)\end{array}$ & & $\begin{array}{c}-0,33 \\
(-0,46 ;-0,19)\end{array}$ \\
\hline Distance/écart initial.e $\times$ & & $-0,34$ & & $-0,33$ \\
\hline 35 ans ou plus & & $(-0,51 ;-0,16)$ & & $(-0,46 ;-0,20)$ \\
\hline$R^{2}$ & 0,02 & 0,20 & 0,12 & 0,35 \\
\hline AIC & 24881 & 24375 & 26685 & 25899 \\
\hline \multicolumn{5}{|c|}{$\begin{array}{l}\text { Lecture : Les modèles A1 et A2 expliquent la distance non genrée entre conjoints (hétérogamie), alors que les } \\
\text { modèles B1 et B2 expliquent l'écart genré en faveur de l'homme (hypergamie féminine). Dans le modèle B2, le } \\
\text { fait que l'homme soit le plus diplômé augmente l'écart en faveur de l'homme à l'enquête de } 6,36 \text { points (sur une } \\
\text { échelle de } 0 \text { à } 100 \text { ) par rapport aux couples de même niveau d'éducation ; dans le même modèle, une augmentation } \\
\text { de l'écart professionnel en faveur de l'homme de } 1 \text { point en début de relation se traduit par une augmentation de } \\
\text { l'écart de } 0,71 \text { point à la date de l'enquête pour les couples formés il y a moins de } 5 \text { ans. } \\
\text { Note : Intervalles de confiance à } 95 \% \text { sous les coefficients. Les coefficients non significatifs au seuil de } 5 \% \\
\text { sont en italique. Variables de contrôle non reprises dans le tableau et dont les effets ne sont pas significatifs } \\
\text { année de naissance du répondant (réf. : } 1948 \text { ), nombre d'enfants dans la relation (aucun; ; } 2 ; 3 \text { ou plus), statut } \\
\text { au moment de l'enquête (actif ; inactif) de chaque conjoint, rang de l'union (première ; ultérieure). La première } \\
\text { modalité listée est prise pour référence. } \\
\text { * Variables centrées (moyenne égale à zéro). } \\
\text { Champ : Relations de couple ou relations amoureuses « importantes » en cours à la date de l'enquête et dans } \\
\text { lesquelles les deux conjoints avaient déjà travaillé au moment de la mise en couple (personnes âgées de } 26 \text { à } 65 \text { ans). } \\
\text { N = } 2817 . \\
\text { Source : Épic (Ined-Insee, 2013-2014). }\end{array}$} \\
\hline
\end{tabular}


contrôle de l'écart genré de départ : + 6 points en faveur de l'homme lorsque celui-ci est plus diplômé, et -6 points dans le cas inverse (les effets sont respectivement de +12 points et -11 points avant contrôle de l'écart professionnel initial).

Dans l'ensemble, l'hypothèse d'un fort effet sur les inégalités économiques entre couples d'un éventuel rapprochement des diplômes des conjoints (Blossfeld et Timm, 2003) est largement invalidée. L'homogamie de diplôme seule ne détermine que faiblement l'écart professionnel en début de relation ou à l'enquête. Certes, l'effet de la différence de diplôme entre conjoints persiste après contrôle de la distance professionnelle de départ, mais avec un apport explicatif et une taille d'effet limités.

\section{Conclusion}

L'allongement des études renforce-t-il l'homogamie lors de la rencontre et tout au long de la vie en couple, comme l'avance la théorie des systèmes éducatifs comme marchés conjugaux (Blossfeld et Timm, 2003) ? La richesse des données françaises récentes issues de l'enquête Épic, qui permet d'associer une analyse des lieux de rencontre et de l'homogamie avec une étude longitudinale des parcours, invite à nuancer cette thèse.

Premièrement, si la proportion de rencontres survenues dans le cadre des études a augmenté au sein des premières unions, passant d'une rencontre sur dix à une sur quatre entre les cohortes nées en 1950 et en 1980, le développement des séparations et des remises en couple a fortement atténué cette tendance pour l'ensemble des relations. Entre ces mêmes cohortes, on passe d'une rencontre sur vingt à une sur dix pour les unions en cours au moment de l'enquête.

Deuxièmement, les relations commencées dans le cadre des études se caractérisent effectivement par une plus forte similarité éducative des conjoints : la moitié des couples qui se sont rencontrés dans ce contexte ont le même niveau de diplôme, contre un tiers en moyenne. Cependant, l'effet sur l'homogamie de l'ensemble des couples - et a fortiori sur les inégalités entre couples - est nécessairement limité, dans la mesure où ces unions restent minoritaires. Ce résultat est cohérent avec l'affaiblissement de l'homogamie de diplôme, de classe sociale et d'origine sociale en France au cours des dernières décennies (Vanderschelden, 2006; Bouchet-Valat, 2014).

Troisièmement, comme le prévoit la théorie des systèmes éducatifs comme marchés conjugaux, la distance entre les positions professionnelles des conjoints est moins grande - de $30 \%$ - lorsqu'ils ont le même diplôme que lorsque l'homme est le plus diplômé. Mais les diplômes ne jouent que faiblement sur la distance professionnelle à la date de l'enquête : un éventuel renforcement de l'homogamie de diplôme n'entraînerait qu'un léger rapprochement des 
situations professionnelles des conjoints et n'aurait donc que peu d'effet sur les inégalités de revenus entre couples. Il apparaît, en outre, qu'une approche genrée (distinguant écart en faveur de l'homme et écart en faveur de la femme) explique mieux la variation des positions professionnelles au sein du couple qu'une approche en termes de distance entre conjoints, qui rend mal compte des différences majeures entre les carrières des hommes et des femmes. Cependant, cette analyse est limitée par l'absence d'informations dans Épic sur la première profession des conjoints qui se sont rencontrés pendant leurs études ou d'éléments sur les relations antérieures à la relation au moment de l'enquête.

Ces résultats indiquent en creux que les déterminants majeurs des inégalités entre ménages sont à rechercher ailleurs que du côté de l'homogamie d'éducation. Ils peuvent tenir à des facteurs économiques, comme la baisse du taux d'emploi des hommes (Breen et Salazar, 2010; Bouchet-Valat, 2017) et la croissance des très hauts revenus (notamment ceux du capital, Landais, 2007). Les facteurs démographiques ont aussi leur importance, en particulier la diminution de la proportion de personnes en couple et le développement des familles monoparentales, dont la part a augmenté parmi les ménages les plus modestes (Bonnet et al., 2017) et qui concernent particulièrement les mères les moins diplômées (Acs et al., 2015). Il semble essentiel de porter plus d'attention à ces phénomènes pour améliorer notre compréhension de l'évolution des inégalités économiques entre ménages.

Remerciements : Les auteurs remercient Vianney Costemalle, Marie Bergström, Arnaud Régnier-Loilier et Wilfried Rault, ainsi que les chercheurs de l'UR3 (Fécondité, familles, conjugalités) de l'Ined, les participants du séminaire café de l'UR9 (Démographie économique) de l'Ined et les rapporteurs anonymes de la revue pour leurs remarques ayant contribué à l'amélioration de ce travail. 


\section{RÉFÉRENCES}

ACS M., LHOMMEAU B., RAYNAUd É., 2015, Les familles monoparentales depuis 1990. Quel contexte familial ? Quelle activité professionnelle ?, Drees, Dossiers solidarité et santé, $\mathrm{n}^{\circ} 67$.

BERGSTRÖM M., 2016, (Se) correspondre en ligne. L'homogamie à l'épreuve des sites de rencontres, Sociétés contemporaines, 104(4), p. 13-40.

Blossfeld H.-P., Timm A. (eds.), 2003, Who Marries Whom? Educational Systems as Marriage Markets in Modern Societies, Dordrecht, Kluwer Academic Publishers.

BONNET C., GARBinti B., GROBON S., 2017, Inégalités d'accès à la propriété et de richesse immobilière au sein des jeunes en France, 1973-2013, Ined, Documents de travail, $\mathrm{n}^{\circ} 234$.

Bouchet-VAlat M., 2014, Les évolutions de l'homogamie de diplôme, de classe et d'origine sociales en France (1969-2011) : ouverture d'ensemble, repli des élites, Revue française de sociologie, 55(3), p. 459-505.

BouChet-VAlat M., 2015, Plus diplômées, moins célibataires. L'inversion de l'hypergamie féminine au fil des cohortes en France, Population, 70(4), p. 705-730.

Bouchet-VAlat M., 2017, Le développement de l'emploi des femmes augmente-t-il les inégalités de salaire entre couples ? Le cas de la France entre 1982 et 2014, Économie et statistique, $\mathrm{n}^{\circ}$ 493, p. 71-90.

Bozon M., HÉRAn F., 1987, La découverte du conjoint : I. Évolution et morphologie des scènes de rencontre, Population, 42(6), p. 943-985.

BOZON M., HÉRAN F., 1988, La découverte du conjoint : II. Les scènes de rencontre dans l'espace social, Population, 43(1), p. 121-150.

Bozon M., Rault W., 2012, De la sexualité au couple. L'espace des rencontres amoureuses pendant la jeunesse, Population, 67(3), p. 453-490.

BREEN R., ANDERSEN S. H., 2012, Educational assortative mating and income inequality in Denmark, Demography, 49(3), p. 867-887.

BREEN R., SALAZAR L., 2010, Has increased women's educational attainment led to greater earnings inequality in the United Kingdom? A multivariate decomposition analysis, European Sociological Review, 26(2), p. 143-157.

BREEN R., SALAZAR L., 2011, Educational assortative mating and earnings inequality in the United States, American Journal of Sociology, 117(3), p. 808-843.

BURRICAND C., GROBON S., 2015, Quels stéréotypes sur le rôle des femmes et des hommes en 2014 ?, Études et résultats, nº 907.

Chambaz C., Maurin É., Torelli C., 1998, Lévaluation sociale des professions en France. Construction et analyse d'une échelle des professions, Revue française de sociologie, 39(1), p. 177-226.

CHAUVEL L., 1998, La seconde explosion scolaire : diffusion des diplômes, structure sociale et valeur des titres, Revue de l'OFCE, n 66, p. 5-36.

COUPPIÉ T., ÉPIPHANE D., 2007, Vivre en couple et être parent : impacts sur les débuts de carrière, Céreq Bref, n 241.

COURTIOUX P., LignON V., 2015, Homogamie éducative et inégalités de revenu salarial : une perspective de cycle de vie, Économie et statistique, n 481-482, p. 149-183. 
Dupray A., Moullet S., 2005, Les salaires des hommes et des femmes. Des progressions particulièrement inégales en début de vie active, Céreq Bref, n 219.

EIKA L., MOGSTAD M., ZAFAR B., 2014, Educational assortative mating and household income inequality, Staff Report $n^{\circ} 682$, Federal Reserve Bank of New York.

FRÉMEAUX N., LEFRANC A., 2015, Assortative mating and earnings inequality in France, Mimeo, centre de recherche Thema, Université de Cergy-Pontoise.

GALLAND O., 1995, Une entrée de plus en plus tardive dans la vie adulte, Économie et statistique, $\mathrm{n}^{\circ}$ 283-284, p. 33-52.

Guichard-Claudic Y., Testenoire A., TRAnCART D., 2009, Distances et proximités conjugales en situation d'homogamie et d'hétérogamie, in Pailhé A., Solaz A. (dir.), Entre famille et travail. Des arrangements de couple aux pratiques des employeurs, Paris, La Découverte, p. 187-207.

KALMiJN M., FlAP H., 2001, Assortative meeting and mating: Unintended consequences of organized settings for partner choices, Social Forces, 79(4), p. 1289-1312.

LANDAIS C., 2007, Les hauts revenus en France (1998-2006) : une explosion des inégalités ?, École d'économie de Paris, Documents de travail.

LAMPARD R., 2007, Couples' places of meeting in late 20th century Britain: class, continuity and change, European Sociological Review, 23(3), p. 357-371.

Laumann E. O., Gagnon J. H., Michael R. T., Michaels S., 1994, The Social Organization of Sexuality: Sexual Practices in the United States, Chicago, University of Chicago Press.

LE Minez S., RouX S., 2001, Les écarts de revenu salarial entre hommes et femmes en début de carrière, Insee première, $\mathrm{n}^{\circ} 801$.

LE Minez S., ROUX S., 2002, Les différences de carrières salariales à partir du premier emploi, Économie et statistique, $\mathrm{n}^{\circ}$ 351, p. 31-63.

MÄENPÄÄ E., JALOVAARA M., 2014, Homogamy in socio-economic background and education, and the dissolution of cohabiting unions, Demographic Research, 30(65), p. 1769-1792.

MAinguené A., MARTinelli D., 2010, Femmes et hommes en début de carrière : les femmes commencent à tirer profit de leur réussite scolaire, Insee première, $\mathrm{n}^{\circ} 1284$.

MARE R. D., 1991, Five decades of educational assortative mating, American Sociological Review, 56(1), p. 15-32.

Meurs D., Pailhé A., Ponthieux S., 2010, Enfants, interruptions d'activité des femmes et écart de salaire entre les sexes, Revue de l'OFCE, 114(3), p. 113-133.

POTARCA G., 2017, Does the internet affect assortative mating? Evidence from the U.S. and Germany, Social Science Research, 61, p. 278-297.

PRIOUX F., 2003, Lâge à la première union en France : une évolution en deux temps, Population, 58(4-5), p. 623-644.

RAULT W., RÉGNIER-LOILIER A., 2015, La première vie en couple : évolutions récentes, Population et sociétés, $\mathrm{n}^{\circ} 521$.

RAULT W., RÉGNIER-LOILIER A., 2019, Étudier les parcours individuels et conjugaux en France. Enjeux scientifiques et choix méthodologiques de l'enquête Épic, Population, 74(1-2), p. 11-40.

Robert-BobéE I., MAZUy M., 2005, Calendriers de constitution des familles et âge de fin des études, in Lefèvre C., Filhon A. (dir.), Histoires de familles, histoires familiales. Les résultats de l'enquête famille de 1999, Paris, Ined, Cahier n 156, p. 175-200.

SCHWARTZ C. R., MARE R. D., 2005, Trends in educational assortative marriage from 1940 to 2003, Demography, 42(4), p. 621-646.

SinGLY (DE) F., 1987, Fortune et infortune de la femme mariée, Paris, Presses universitaires de France. 
TESTENOIRE A., 2008, Carrières féminines, résistances masculines : couples à hypogamie féminine, in Guichard-Claudic Y., Kergoat D., Vilbrod A. (dir.), L’inversion du genre. Quand les métiers masculins se conjuguent au féminin... et réciproquement, Rennes, Presses universitaires de Rennes, p. 385-396.

VANDERSCHELDEN M., 2006, Homogamie socioprofessionnelle et ressemblance en termes de niveau d'études : constat et évolution au fil des cohortes d'unions, Économie et statistique, $\mathrm{n}^{\circ}$ 398-399, p. 33-58.

VillenEUVE-GOKALP C., 1990, Du mariage aux unions sans papiers : histoire récente des transformations conjugales, Population, 45(2), p. 265-297.

WORNER S. M., 2006, The effects of assortative mating on income inequality: A decompositional analysis, CEPR Discussion Paper $n^{\circ} 538$, Canberra, Centre for Economic Policy Research, Research School of Economics, Australian National University. 


\section{Milan Bouchet-VAlat, Sébastien GRobon • Homogames Un Jour, homogames TOUJOURS? RENCONTRE PENDANT LES ÉTUDES ET PROXIMITÉ DE DIPLÔME ET DE CARRIÈRE AU SEIN DES COUPLES EN FRANCE}

Cet article analyse, à partir de l'enquête Étude des parcours individuels et conjugaux (Épic, Ined-Insee, 2013-2014) réalisée en France, le lien entre le cadre de rencontre des conjoints, leurs diplômes et leurs carrières professionnelles. II remet en cause la thèse selon laquelle l'allongement des études renforcerait leur proximité éducative et socio-économique, aggravant ainsi les inégalités entre couples dans l'ensemble de la population. Au fil des cohortes, la proportion de premières rencontres survenues dans le cadre des études ou pendant ces dernières s'est accrue, mais les séparations et remises en couple atténuent fortement les conséquences de cette évolution pour les relations en cours au moment de l'enquête. Les couples formés dans le cadre des études se caractérisent, comme on pouvait l'attendre, par une plus forte similarité des diplômes des conjoints. Cette homogamie éducative favorise une homogamie de statut professionnel, au début de la relation, comme à la date de l'enquête, mais ce lien est assez faible. Et le fait de s'être rencontrés dans le cadre des études ne joue que de façon très limitée sur la proximité des positions professionnelles des conjoints. Les inégalités genrées (hypergamie) apparaissent finalement plus fortes que l'homogamie : ce n'est pas lorsqu'ils ont le même diplôme, mais lorsque la femme est la plus diplômée, que l'écart professionnel en faveur de l'homme est le plus faible.

\section{Milan Bouchet-Valat, Sébastien Grobon • Once homogamous, Always Homogamous? Educational LeVel and Career Similarity of Couples in France Who MeEt AT SCHOOL}

Drawing on data from the EPIC study of individual and conjugal trajectories (Étude des parcours individuels et conjugaux, INED-INSEE, 2013-2014, France), this article analyses the context in which couples met, the level of education they reached, and their subsequent careers. It calls into question the claim that longer education bolsters partners' educational and socioeconomic similarity and exacerbates intercouple inequality in the population at large. The proportion of couples who meet in an educational context has risen across birth cohorts. However, for relationships that were in progress at the time of the survey, separations and repartnering greatly attenuate the impact of this increase. As expected, partners who met at school show very similar educational levels. But while educational homogamy works in favour of occupational status homogamy at both the time the relationship started as well as at the time of the survey, that tie is quite weak. Having met at school only slightly affects the difference between partners' occupational positions. Gender inequalities (hypergamy) ultimately appear stronger than homogamy: it is in couples where the woman's level of education is higher than the man's that the occupational gap in the man's favour is smallest.

\section{Milan Bouchet-VALAT, Sébastien GROBOn • ¿Homógamos un día, homógamos PARA SIEMPRE? ENCUENTRO DURANTE LOS ESTUDIOS Y PROXIMIDAD DE DIPLOMA Y DE CARRERA EN EL SENO DE LAS PAREJAS EN FRANCIA}

A partir de la encuesta Epic Estudio de las trayectorias individuales y conyugales (Étude des parcours individuels et conjugaux, (Ined-Insee, 2013-2014, Francia), este artículo analiza la relación entre el marco del encuentro de los cónyuges, sus diplomas y sus carreras profesionales. Cuestiona la hipótesis según la cual el alargamiento de los estudios reforzaría la proximidad educativa y socio-económica de dichos cónyuges, agravando así las desigualdades entre las parejas en el conjunto de la población. Siguiendo las cohortes, la proporción de primeros encuentros en el marco de los estudios o durante estos, ha aumentado pero las separaciones y los retornos a la vida de pareja atenúan fuertemente las consecuencias de esta evolución sobre las relaciones en curso en el momento de la encuesta. Las parejas formadas en el marco de los estudios se caracterizan, como se podía suponer, por una mayor similitud en los diplomas de ambos cónyuges. Esta homogamia educativa favorece una homogamia de estatus profesional, tanto en los comienzos de la relación como en el momento de la encuesta, pero esta relación es floja. Y el hecho de haberse encontrado en el marco de los estudios, juega de manera muy limitada sobre la proximidad de las posiciones profesionales de los cónyuges. Las desigualdades determinadas de género (hipergamia) aparecen finalmente más fuertes que la homogamia: no es cuando ellos tienen el mismo diploma que ellas, sino cuando la mujer es más diplomada, que la diferencia profesional a favor del hombre es la más insignificante.

Mots-clés : Épic, couple, homogamie, hypergamie, cadre de rencontre, scolarisation, inégalités femmes-hommes, carrières professionnelles, genre, France

Keywords: EPIC survey, couple, homogamy, hypergamy, meeting place, education, gender inequalities, occupational careers, gender, France 
